Molecular Biology Laboratory), and PIR.

\section{Specialized Programs}

Several special-purpose programs are also hitting the market. For example, researchers at the University of Delaware (Newark) and HewlettPackard (Palo Alto, CA) have produced a microbial identification system for medical, academic, and industrial laboratories. The system automatically characterizes unknown bacteria via gas chromatographic analysis of the cells' fatty acids. Operating software, priced at $\$ 3,000$, is marketed by Microbial ID, Inc. (Newark, DE).

In this process, the bacterial fatty acids are chemically converted to the corresponding methyl ester. Chromatographic data are analyzed by the computer and printed out. The microbes are identified by matching flame ionization spectra to computerized libraries of about 140 anaerobic species and more than 300 aerobic species; priced at $\$ 3,000$ each, the libraries are updated every six months. Since fatty acid composition is a stable and specific genetic trait, the process could replace other biochemical methods that often rely on analyst training and subjective judgments, explains Myron Sasser, professor of plant science at the University and author of the aerobic library.

So-called expert systems are also finding their way into the biolaboratory. Basically, the programs consist of a series of decision-making algorithms (the shell) and hundreds or thousands of if/then rules (the knowledge base), defined by an experienced user, on which the shell operates. By comparing experimental or process data with the knowledge base, the system alerts the operator to trouble spots and inconsistencies and "recommends" alternatives.

One example is Strategene, developed by IntelliGenetics (Mountain View, CA). Based on the company's KEE (Knowledge Engineering Environment) shell, Strategene helps evaluate cloning techniques by organizing nucleic acid data. The system includes a large knowledge base of nucleic acids and commercially available plasmids, complete with size, sequence, history, and restriction maps.

Like other commercially available expert systems, Strategene was orginally designed for use on relatively large, expensive workstations. However, the company is now developing $\mathrm{PC}$ versions.

For a free copy of this article (while available), write in 506 on the Reader Service Card

BIOIECH SOFWARE

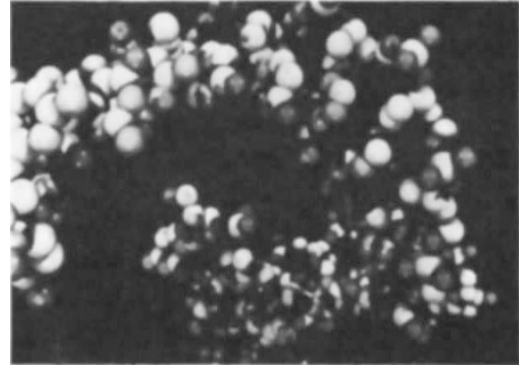

High-level graphics software. Dynamic Object-Rendering Environment (DORÉ) software from Ardent Computer Corp. (Sunnyvale, CA) allows interactive visualization of complex data generated by supercomputer-class applications. It is the first package to integrate the computation and analysis of complex data with advanced graphics. The software library lets users describe a scene, produce highly complex images from the scene data, and manipulate the data interactively and dynamically. The company is offering source-code licenses to universities and research labs for a nominal charge $(\$ 250)$, prior to the package's release in July 1988; licenses are also available to commercial users for a $\$ 15,000$ fee.

Write in 801 on Reader Service Card

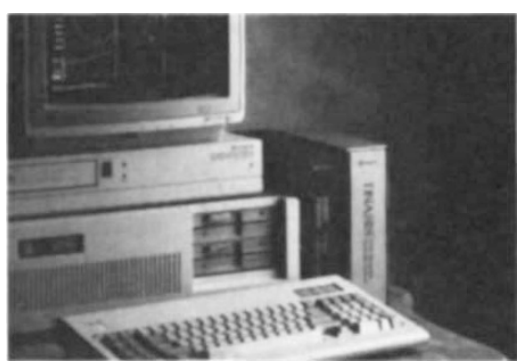

Protein sequence analysis is facilitated with HIBIO PROSIS from $\mathrm{Hi}$ tachi (San Bruno, CA). Key features in the program include secondary structure predictions; amino acid maximum homology between two sequences; amino acid conversion, composition/molecular weight, and homology search; hydrophobicity analysis; homology plot; and keyword search and database access functions. The AMIEDIT phase of the program creates or edits amino acid sequence files; data may be keyed in or entered by digitizer and voice feedback. The PROTES phase performs analyses on sequences and displays the results graphically. DBREF searches the NBRF-PIR database for a target sequence, and can perform homology searches. The company's DNASIS program, for DNA sequence analysis, has been improved to achieve faster homology searches. Write in 804 on Reader Service Card

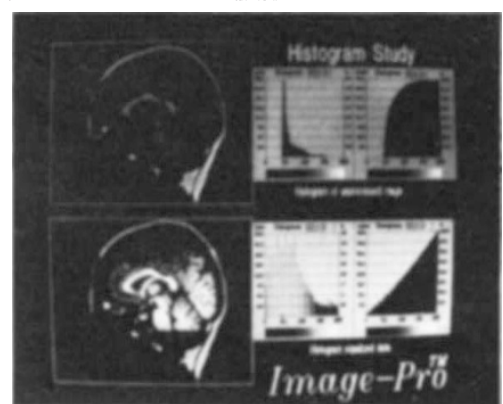

Image-processing software. Media Cybernetics (Silver Spring, MD) announces the release of Image-Pro II, a new version of its powerful imageprocessing software. A new interface allows the user to select a single monitor configuration with pull-down menus on the display monitor or a dual-monitor configuration in which the menus appear on the system monitor. A free-form feature allows processing on " $n$ vertex polygonal areas" which users specify by tracing the area desired; an automatic preference feature provides for maintenance of global environmental settings from one session to the next. The accompanying manual includes diagrams, illustrations, a tutorial, and a chapter on image-processing theory and terms. A variety of input and output devices allow camera-, film recorder-, and printer-interchangeability. Versions are compatible with products offered by major hardware vendors including Data Translation, Imagraph, AT\& $\mathrm{T}$, and more.

Write in 802 on Reader Service Card

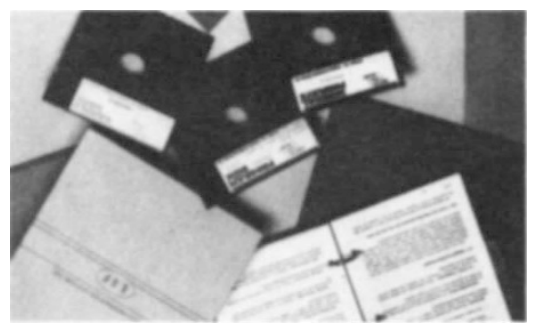

Software for publishing scientists. Reference Manager ${ }^{\mathrm{TM}}$ is a microcomputer-based software package designed specifically for publishing scientists. A specialized database management program combined with a text-reformatting module, the package stores bibliographic references that may later be incorporated into manuscripts and used to create bibliographies. The recently updated Version 4.0 is compatible with IBM/PCs, ATs, XTs, and PS/2s, and may be used with most word processing systems; a version for use with Macintosh computers is newly available as well.

Write in 807 on Reader Service Card 